\title{
Approach to chest pain and acute myocardial infarction
}

\author{
S Pandie, ${ }^{1}$ FCP (SA), Cert Cardiology (SA); D Hellenberg, ${ }^{2}$ MFamMed, FCFP (SA); F Hellig, ${ }^{3}$ BSc, MB BCh, FCP (SA), FSCAI; \\ M Ntsekhe, ${ }^{1} \mathrm{MD}, \mathrm{PhD}$ \\ ${ }^{1}$ Division of Cardiology, Department of Medicine, Faculty of Health Sciences, University of Cape Town and Groote Schuur Hospital, \\ Cape Town, South Africa \\ ${ }^{2}$ Division of Family Medicine, School of Public Health and Family Medicine, Faculty of Health Sciences, University of Cape Town, South Africa \\ ${ }^{3}$ Sunninghill and Sunward Park Hospitals, Johannesburg, South Africa
}

Corresponding author: S Pandie (s.pandie@uct.ac.za)

\begin{abstract}
Patient history, physical examination, 12-lead electrocardiogram (ECG) and cardiac biomarkers are key components of an effective chest pain assessment. The first priority is excluding serious chest pain syndromes, namely acute coronary syndromes (ACSs), aortic dissection, pulmonary embolism, cardiac tamponade and tension pneumothorax. On history, the mnemonic SOCRATES (Site Onset Character Radiation Association Time Exacerbating/relieving factor and Severity) helps differentiate cardiac from non-cardiac pain. On examination, evaluation of vital signs, evidence of murmurs, rubs, heart failure, tension pneumothoraces and chest infections are important. A 12-lead ECG should be interpreted within 10 minutes of first medical contact, specifically to identify ST elevation myocardial infarction (STEMI). High-sensitivity troponins improve the rapid rule-out of myocardial infarction (MI) and confirmation of non-ST elevation MI (NSTEMI). ACS (STEMI and NSTEMI/unstable angina pectoris (UAP)) result from acute destabilisation of coronary atheroma with resultant complete (STEMI) or subtotal (NSTEMI/UAP) thrombotic coronary occlusion. The management of STEMI patients includes providing urgent reperfusion: primary percutaneous coronary intervention (PPCI) if available, deliverable within 60 - 120 minutes, and fibrinolysis if PPCI is not available. Essential adjunctive therapies include antiplatelet therapy (aspirin, $\mathrm{P}_{12} \mathrm{Y}_{12}$ inhibitors), anticoagulation (heparin or low-molecular-weight heparin) and cardiac monitoring.
\end{abstract}

S Afr Med J 2016;106(3):239-245. DOI:10.7196/SAMJ.2016.v106i3.10323

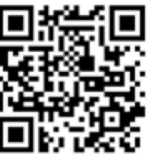

Chest pain is a common cause for attendance at emergency and primary care consultation rooms. The lifetime prevalence accounts for 20 - $40 \%$ of the general population, with an incidence of $0.7 \%$ in primary care. ${ }^{[1]}$ The majority of these presentations do not constitute a life-threatening illness, and the most common causes are musculoskeletal, respiratory, gastrointestinal and psychogenic. The challenge to the primary attending clinician is to rapidly and accurately diagnose potentially serious acute chest pain syndromes (Table 1), before turning attention to acute and chronic conditions that may also carry risk for mortality and morbidity, e.g. pneumonia, stable coronary artery disease $(\mathrm{CAD})$, pericarditis, valvular heart disease, lung malignancies and abdominal pathologies. Finally, less serious conditions need consideration. At primary care level, clinicians need to identify serious conditions reliably while not exposing patients to unnecessary investigations and hospital admissions. ${ }^{[2]}$ This review focuses on acute coronary syndromes (ACSs), which are the most common of the life-threatening chest pain presentations.

A clear stepwise approach to acute chest pain is critical to avoid misdiagnoses (Fig. 1). In the absence of chest trauma, the initial assessment should focus on ACS (acute myocardial infarction (MI) or unstable angina), aortic dissection, pulmonary embolism, cardiac tamponade, tension pneumothorax and, less commonly, acute abdominal syndromes presenting as chest pain. The patient's history and risk factor profile, along with the clinical assessment, should direct the clinician to the next most appropriate diagnostic and therapeutic intervention.

\section{Emergency chest pain assessment}

\section{General considerations}

Most chest pain algorithms aim to exclude ACSs. Initial and continuous assessment of vital signs (blood pressure (BP), pulse, cardiac monitoring and oxygen saturations) should be standard care. If the patient is tachypnoeic or has oxygen saturations $<94 \%$, supplemental oxygen should be administered. Pain should be managed with appropriate analgesia, preferably intravenous opioids such as morphine. A focused history, thorough physical examination, meti- culous review of a 12-lead electrocardiogram (ECG) and appropriately timed measurement of cardiac biomarkers are the key components of an effective chest pain assessment.

\section{History}

The history is the most useful component when evaluating chest pain, as both normal and abnormal investigations (such as the ECG, cardiac biomarkers and chest radiograph (CXR)) must be interpreted in the context of the patient's history.

The mnemonic SOCRATES (Site Onset Character Radiation Association Time Exacerbating/relieving factor and Severity) helps differentiate cardiac v. non-cardiac (respiratory, gastric or musculoskeletal) pain. Features that make a diagnosis of ACS likely include: central (retrosternal) location; sudden or acute onset; heavy or burning sensation with radiation to the arm or jaw; associated dyspnoea, nausea or sweating; duration > 15 minutes; relief of symptoms by nitrates; worsening of symptoms by activity; chest or left-arm discomfort that is the same in nature as previously documented angina; and a known history of CAD (including prior MI) ${ }^{[3]}$ Atypical presentations need to be considered in the elderly, diabetics and women.

At the primary care level, the Marburg Heart Score has been shown to have excellent negative predictive value (Table 2). Based only on variables acquired on history and examination, it is an easy-to-apply clinical prediction rule. ${ }^{[4]}$

\section{Physical examination}

All patients presenting with acute chest pain should undergo a thorough physical examination. In ACS, the cardiac examination is often unremarkable, but specific attention should be paid to the vital signs (pulse and BP), features of heart failure, and auscultation precordial murmurs and rubs. The presence of transient mitral regurgitation, hypotension, diaphoresis, pulmonary oedema, crackles or signs of extracardiac vascular disease is highly suggestive of ACS. ACS is less likely if the presenting chest pain is reproducible by chest palpation. ${ }^{[3]}$ Examination of the respiratory system should exclude 


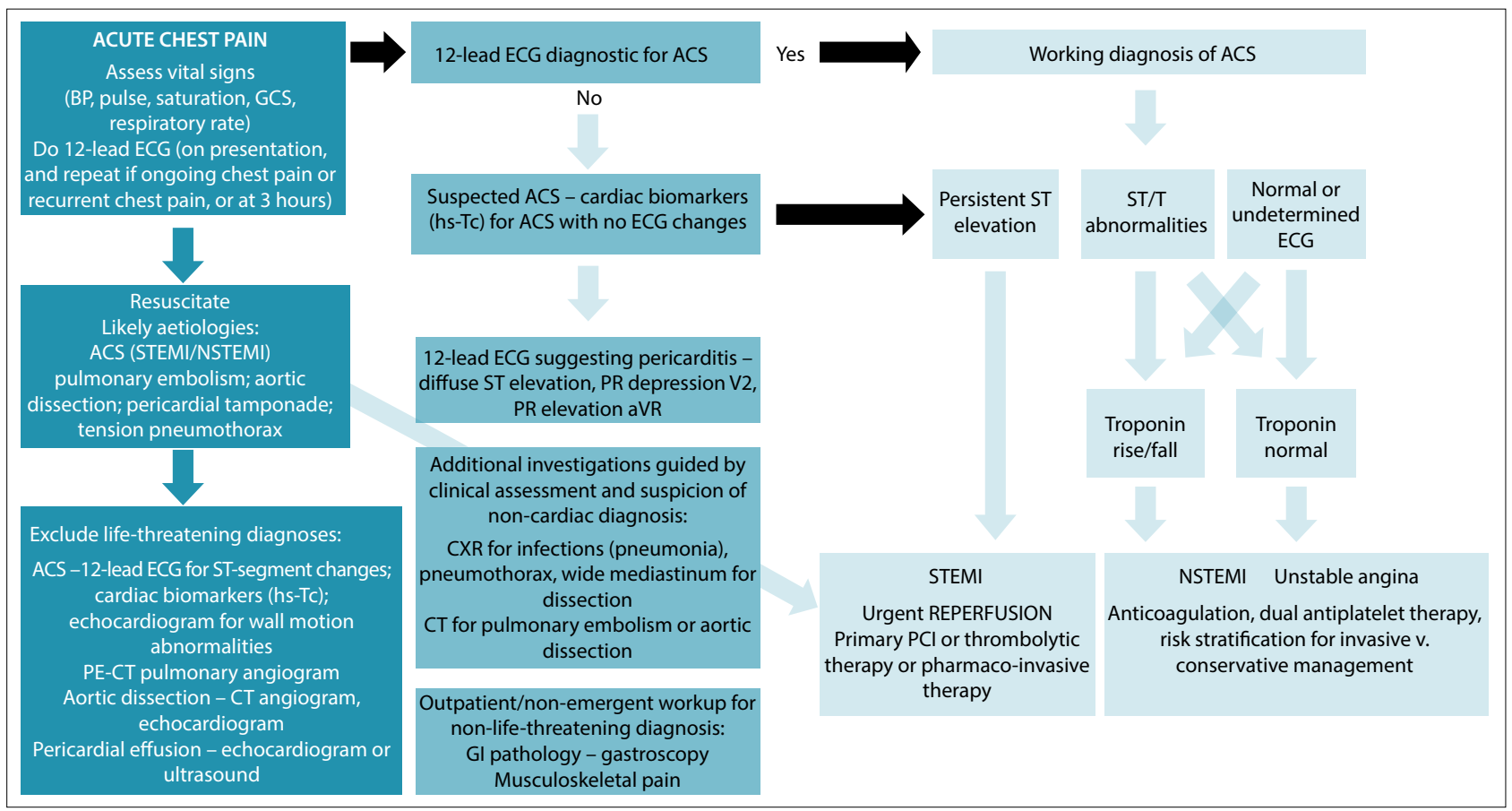

Fig. 1. Chest pain algorithm $(B P=$ blood pressure; $G C S=$ Glasgow coma scale; $A C S=$ acute coronary syndrome; $h$ s-Tc = high-sensitivity troponin; $G I=$ gastrointestinal $P C I=$ percutaneous coronary intervention; $P E-C T=$ pulmonary embolism-computed tomography; STEMI = ST elevation myocardial infarction; NSTEMI = non-ST elevation myocardial infarction).

tension pneumothoraces and chest infections, while abdominal examination may reveal abdominal aetiologies.

\section{2-lead ECG}

Patients presenting with acute chest pain should have a 12-lead ECG performed and interpreted within 10 minutes of first medical contact. If the initial ECG is non-diagnostic but the diagnosis of ACS is highly suspected, serial ECGs should be performed (at 3 hours, 6 hours, 9 hours and 24 hours, or on recurrence of symptoms), including ECGs with additional leads for evaluation of the posterior-lateral myocardial wall V7 V8 V9 or V3R V4R V5R. ACS diagnostic features on the ECG include ST elevation, ST depression, T-wave inversion, and new or presumed new left bundle branch block. ECG features suggesting pericarditis include saddle-shaped ST elevation not confined to an anatomical location, with PR depression in V2 and PR elevation in aVR. ECG features suggestive of pulmonary embolism include tachycardia, features of right ventricular strain (T-wave inversion V1 - V3 with either right axis deviation or tall $\mathrm{R}$ in $\mathrm{V} 1$ ) and the typical SIQIIITIII pattern.

\section{Cardiac biomarkers}

Cardiac biomarkers reflect myocardial cell injury, the hallmark of MI. Pathophysiologically, MI results from ischaemia sufficient to cause cell death, with the release of cellular proteins and enzymes. Cardiac troponins I and $\mathrm{T}$ have been shown to be the most sensitive indicators of cardiac injury, as well as being predictors for short- and long-term outcomes as regards death and MI. ${ }^{[5]}$ The introduction of high-sensitivity troponin (hs- $\mathrm{Tc}$ ) assays have improved rapid ruleout MI, and the confirmation of non-ST elevation MI (NSTEMI). ${ }^{[6]}$ It is important to understand that the increased sensitivity of the test results in lower specificity. To this end, the universal classification of MI was revised in 2012, acknowledging that for practical purposes, the diagnosis of ST elevation MI (STEMI) is made in the correct clinical setting with the appropriate ECG changes, and that MI without ST elevation (NSTEMI) requires positive biomarkers (Table 3). If serial biomarkers are negative, this implies no MI has occurred, but the

\author{
Table 1. Differential for acute chest pain \\ Cardiovascular \\ Coronary artery disease \\ Acute coronary syndromes \\ Angina due to demand/supply mismatch \\ Chronic stable angina \\ Acutely increased myocardial oxygen demand \\ Coronary vasospasm, 'variant angina' \\ Cardiac syndrome $\mathrm{X}$ \\ Coronary artery dissection \\ Coronary anatomic anomalies \\ Valvular heart disease \\ Aortic dissection \\ Pericarditis \\ Myocarditis \\ Stress-induced cardiomyopathy \\ Acute aortic syndromes \\ Chest pain related to hyperadrenergic states \\ Cocaine intoxication \\ Amphetamine intoxication \\ Phaeochromocytoma
}

Continued ...

patient may still require treatment for unstable angina. In addition, positive biomarkers may reflect myocardial injury secondary to noncoronary causes - usually as a result of imbalance between myocardial oxygen supply and demand - and should therefore be interpreted in the clinical context of the presentation. ${ }^{[7]}$

\section{CXR}

The CXR is helpful when pneumothorax or pneumonia, aortic dissection (wide mediastinum) or pulmonary embolism (oligaemia of lung fields or wedge-shaped infarcts) is suspected. If, on the 


\section{Table 1. (continued) Differential for acute chest pain}

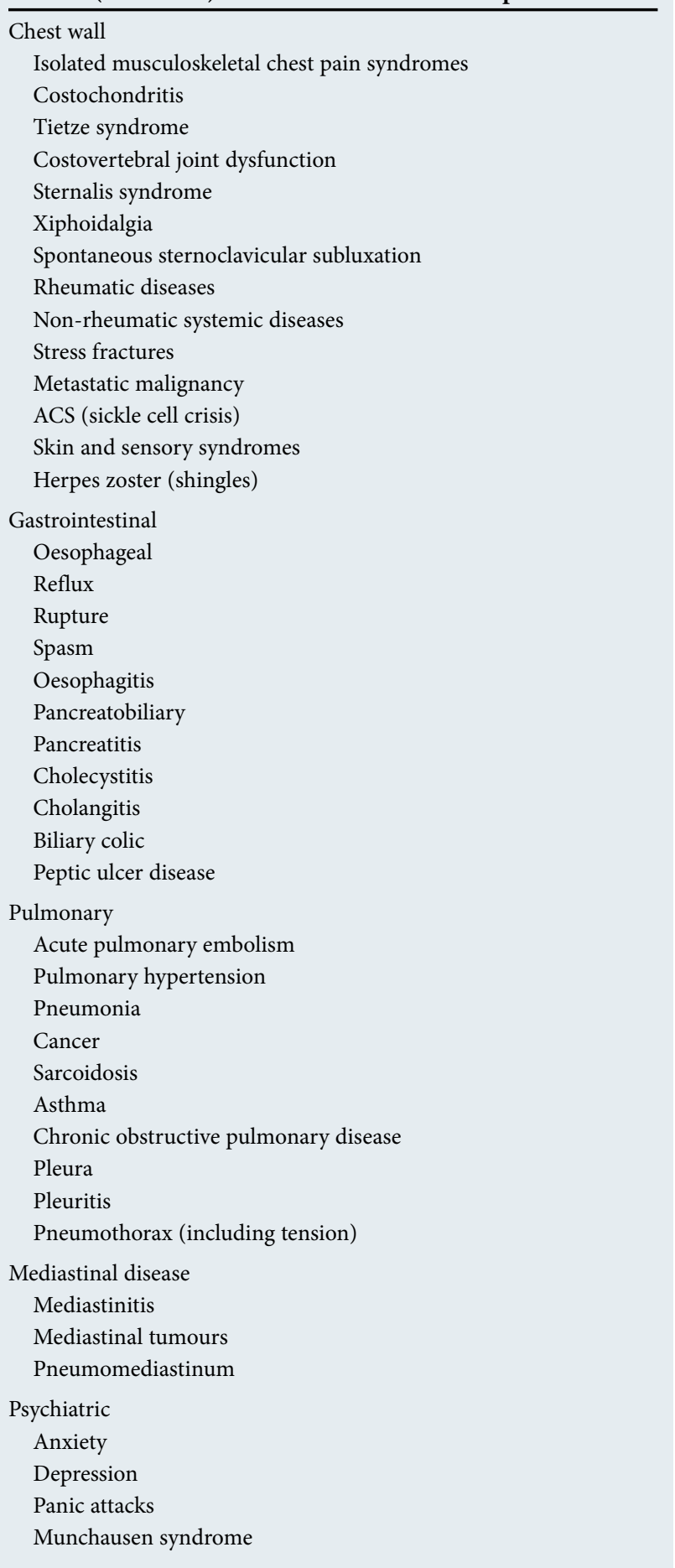

basis of the ECG, ACS is suspected, the urgency of obtaining a CXR should be relegated and implementation of management plans prioritised.

\section{The management of $\mathrm{ACS}$}

The clinical presentations of CAD include stable angina pectoris, silent ischaemia, unstable angina, MI, heart failure and sudden death. ${ }^{[8]}$ ACSs result from acute destabilisation of coronary atheroma with resultant complete or subtotal thrombotic coronary occlusion. Ischaemic discomfort or chest pain is experienced because of reduction in blood flow to the myocardium.
Table 2. The Marburg Heart Score

\begin{tabular}{ll}
\hline Criteria & Score \\
\hline Female $>64$ years; male $>54$ years & 1 \\
$\begin{array}{l}\text { Known CAD, cerebrovascular disease or peripheral } \\
\text { vascular disease }\end{array}$ & 1 \\
Pain worse with exercise & 1 \\
Pain not reproducible with palpation & 1 \\
$\begin{array}{l}\text { Patient assumes pain is cardiac } \\
\text { Interpretation }\end{array}$ & 1 \\
$\quad 97 \%$ of patients with a score of $\leq 2$ will not have CAD & \\
$\sim 23 \%$ of patients with a score of $>3$ will have CAD &
\end{tabular}

\section{STEMI}

Pathophysiology and initial diagnosis

Plaque disruption exposes thrombogenic substances, resulting in intracoronary thrombus formation. Local anticoagulant production is increased and, if successful, will result in thrombus resolution and arrest of the infarction. However, if complete arterial occlusion occurs, myocardial ischaemia followed by infarction results, producing the typical ST elevation seen on the 12-lead ECG. Diagnostic patterns of ST elevation vary depending on which epicardial vessel is involved (Fig. 2).

\section{Immediate management}

The optimal management of STEMI patients includes providing the fastest, safest, most effective method of reperfusion therapy available, i.e. primary percutaneous coronary intervention (PPCI) or fibrinolysis, while simultaneously initiating essential adjunct therpies and monitoring (Fig. 3). Unless contraindicated, all patients should receive antiplatelet therapy (aspirin with $\mathrm{P}_{2} \mathrm{Y}_{12}$ inhibitors such as ticagrelor, prasugrel or clopidogrel) and anticoagulation (unfractionated or low-molecular-weight heparins (LMWHs) dosed to body weight). Patients should be connected to a cardiac monitor capable of cardioversion and transcutaneous pacing. Oxygen should be administered if the patient is hypoxic, and opioids for pain relief, breathlessness and anxiety are essential. ${ }^{[9]}$

\section{Prehospital logistics of care}

The lack of a formal STEMI referral network for both public and private sectors makes decisions regarding reperfusion therapy challenging, and often impedes the rapid administration of reperfusion therapy. ${ }^{[10]}$ Important timeframes that govern timely STEMI care are outlined in Fig. 4. Prehospital logistics and patientpractitioner education should aim to reduce delays from time of symptom onset to definitive reperfusion. ${ }^{[9,11]}$

\section{Reperfusion therapy}

This includes PPCI, fibrinolysis and pharmaco-invasive strategies. Selecting the most appropriate reperfusion strategy depends on three key factors: the time delay from symptom onset to first medical contact; availability; and clinical factors that may favour one strategy over the other.

\section{Patients presenting within 12 hours of onset of symptoms (or since worse exacerbation)}

As a general guide, STEMI patients presenting to PCI-capable facilities should be offered PPCI (preferably within 60 minutes). If there is an anticipated delay of $>120$ minutes from the time of first medical contact, fibrinolysis should be administered. Patients presenting to 
Table 3. Universal classification of MI (adapted from Thygesen et al. ${ }^{[7]}$ )

\begin{tabular}{ll}
\hline Type & Description \\
\hline Type 1: Spontaneous MI & $\begin{array}{l}\text { Related to atherosclerotic plaque rupture, ulceration, erosion or dissection with resultant coronary luminal } \\
\text { thrombus in one or more coronaries, leading to decreased myocardial blood flow with ensuing myocyte necrosis. } \\
\text { Patients may have underlying CAD (severe obstructive or mild non-obstructive) or no CAD. }\end{array}$ \\
$\begin{array}{ll}\text { Type 2: MI secondary to } \\
\text { an ischaemic imbalance }\end{array}$ & $\begin{array}{l}\text { Myocardial injury with necrosis secondary to an imbalance between myocardial oxygen supply and demand. Patients } \\
\text { may or may not have underlying CAD. Common examples include coronary endothelial dysfunction, coronary artery } \\
\text { spasm, tachycardias, bradycardias, anaemia, respiratory failure, hypotension and hypertension (with or without left } \\
\text { ventricular hypertrophy). }\end{array}$ \\
$\begin{array}{l}\text { Type 3: MI resulting in death } \\
\text { when biomarker values are } \\
\text { unavailable, or at autopsy } \\
\begin{array}{l}\text { Types 4a and 4b: } \\
\text { MI related to PCI }\end{array}\end{array}$ & $\begin{array}{l}\text { PCI-related infarction with elevation in cardiac troponins, symptoms of myocardial ischaemia, new ECG changes, } \\
\text { angiographic changes (loss of vessel patency or new wall motion abnormality), or stent thrombosis. }\end{array}$ \\
$\begin{array}{l}\text { Type 5: MI related to } \\
\text { CABG }\end{array}$ & $\begin{array}{l}\text { CABG-related infarction with elevation in cardiac troponins, symptoms of myocardial ischaemia, new ECG } \\
\text { changes, angiographic changes (new graft or native coronary occlusion or new wall motion abnormality). }\end{array}$ \\
PCI = percutaneous coronary interventions; CABG = coronary artery bypass grafting.
\end{tabular}

non-PCI-capable facilities should be considered for fibrinolysis, or transfer for PPCI if PCI can be ensured within 120 minutes. It is important to remember that PPCI and fibrinolysis are of equivalent efficacy for patients presenting within 3 hours of symptoms, and every effort should be made not to delay reperfusion. ${ }^{[12]}$

\section{Fibrinolysis and pharmaco-invasive strategies}

In most sub-Saharan countries, access to PPCI-capable facilities is limited. Fibrinolysis is therefore the first choice reperfusion therapy. Clear contraindications to fibrinolysis need to be identified during the initial assessment (Table 4). Alteplase (tPA) with concomitant heparin was shown to be more effective than streptokinase (one fewer death per 100 patients treated, at the expense of three additional strokes). Tenectaplase is equivalent to accelerated tPA for 30-day mortality (with lower rate of bleeding). ${ }^{[13,14]}$ Non-patient-related factors when considering choice of fibrinolysis include cost, ease of administration and administration in hospital or before being admitted.

Pharmaco-invasive management refers to the combination of fibrinolysis and angiography, with a view to PCI of the culprit lesion. Patients who have had successful fibrinolysis can be referred to a PCIcapable centre for routine angiography and PCI within 24 hours of completing fibrinolysis. This strategy has been shown to reduce recurrent MI and urgent revascularisation for recurrent ACSs, and is currently a very attractive option in South African (SA) clinical settings. ${ }^{[15]}$

\section{Patients presenting after 12 hours of symptom onset (or since worse exacerbation)}

Beyond 12 hours, fibrinolysis is far less effective than PPCI, while still carrying the risk of bleeding. Decisions regarding reperfusion should therefore be on an individual basis. Patients with persistent myocardial ischaemia, hypotension, haemodynamic instability or ventricular arrhythmias should be considered for referral for angiography and PCI. Stable patients should receive antiplatelet therapy and anticoagulation with unfractionated heparins or LMWHs. ${ }^{[12]}$

\section{Failed fibrinolysis}

The 12-lead ECG should be repeated 60 (tPA) - 90 (streptokinase) minutes after fibrinolysis to assess therapeutic success. Failed fibrinolysis is indicated by failure of the ST elevation to reduce by $>50 \%$ with or without persistent myocardial ischaemia. These patients do not benefit from repeat fibrinolysis, and should be considered for urgent rescue PCI, ideally within 12 hours. ${ }^{[12]}$

\section{NSTEMI and unstable angina}

\section{Pathophysiology and initial diagnosis}

The pathophysiology of most NSTEMIs involves the rupture of a vulnerable atheromatous plaque with subsequent thrombus formation, leading to reduced blood flow and ischaemia, with or without MI (NSTEMI or unstable angina (UA), respectively).

The diagnosis is confirmed by the presence of prolonged ( $>20$ minutes) ischaemic chest pain, new-onset Canadian Class II or III angina, acute deterioration of stable chronic angina or angina following a recent MI, and the absence of ST elevation on the 12-lead ECG. Atypical complaints (including silent ischaemia, epigastric pain, indigestion and dyspnoea) can occur in older patients ( $>75$ years), women and patients with diabetes, chronic kidney disease or dementia. In these settings, ST-T wave abnormalities on ECG should prompt rapid rule-out or rule-in of ACS using hs-Tc assays (Fig. 5).

\section{Immediate management and risk stratification}

The immediate care of UA and NSTEMI patients involves two key steps: (i) risk stratification using validated ischaemia and bleeding prediction tools before deciding on pharmacological and invasive management strategies; and (ii) the administration of evidence-based therapies. If there are no contraindications, a patient with a suspected diagnosis of ACS should receive antiplatelet therapy (aspirin and clopidogrel), anticoagulation therapy (unfractionated heparins or LMWHs, or indirect factor Xa inhibitors (fondaparinux)), and anti-ischaemic agents ( $\beta$-blockers, or calcium channel blockers and nitrates).

Validated ischaemic risk prediction scores (Thrombolysis in MI (TIMI) Risk Score and the Global Registry of Acute Coronary Events (GRACE) Risk Score) use demographic, clinical, ECG and biochemical variables to estimate the short-, intermediate- and longterm prognosis related to the current presentation (Table 5). The validated CRUSADE (Can Rapid risk stratification of Unstable angina patients Suppress ADverse outcomes with Early implementation of ACC/AHA guidelines)bleeding score combines eight predictors of major bleeding, allowing a physician to estimate the baseline risk of in-hospital major bleeding, therefore complementing ischaemic risk prediction tools. These scores are readily available as applications on smartphones and tablets, making them easy to use in daily practice. Patients with intermediate- to high-risk scores should be considered for invasive management, and advanced for coronary angiography with a view to revascularisation. Independent of risk 




Fig. 2. ST elevation MI variations. scores, patients with significant rise or fall of troponin levels, dynamic ECG changes and ongoing ischaemia should be considered for invasive management.

\section{Timing of angiography and intervention} High-risk patients benefit from revascularisation therapy. Patients with ongoing ischaemia, acute heart failure, life-threatening ventricular arrhythmias or haemodynamic instability should be considered for immediate intervention regardless of biomarkers and ECG changes. Patients with high-risk scores who have clinically stabilised should be considered for angiography within 48 hours of admission. Low-risk patients may be managed medically, or have coronary angiography deferred. These timelines are a guide and may differ based on the availability of PCIcapable centres.

\section{Hospitalisation and discharge}

All ACS patients should be managed in a high-care setting, specifically for the rapid identification and management of haemodynamic (cardiogenic shock) and arrhythmic (ventricular tachycardia and fibrillation) complications, as well as bleeding (access site or secondary to fibrinolysis) and recurrent infarction. ECG monitoring and bed rest should continue for at least 24 hours after successful reperfusion and symptom resolution.

Chronic medications that offer mortality benefit should be introduced while in hospital (unless contraindicated). Dual antiplatelet therapy should be continued for 1 year, unless there are specific reasons to stop sooner. The introduction of $\beta$-blockers, renin-angiotensin system inhibitors, angiotensin-converting enzyme inhibitors (ACE-Is), angiotensin receptor blockers (ARBs) or aldosterone antagonists) and statins should be specific to the individual, rather than introduced as a routine 'cocktail'. The early introduction of high-dose HMG-CoA reductase inhibitors (statins) is also associated with improved survival and reduced morbidity. Betablockers should be provided to all patients within 24 hours unless there is evidence of haemodynamic compromise, congestive heart failure or brady-arrhythmias (such as heart block). Patients with anterior MI, evidence of heart failure or depressed left ventricular systolic function should receive ACE-Is or ARBs, plus an aldosterone antagonist. Lifestyle and risk factor modification including smoking cessation, diet, physical activity and BP control is essential. ${ }^{[9]}$ Patients who require surgical revascularisation should be discussed with a combined heart team (interventional cardiologist, clinical cardiologist and cardiothoracic surgeon) regarding the optimal timing of surgery (in-patient $\mathrm{v}$. elective re-admission). 


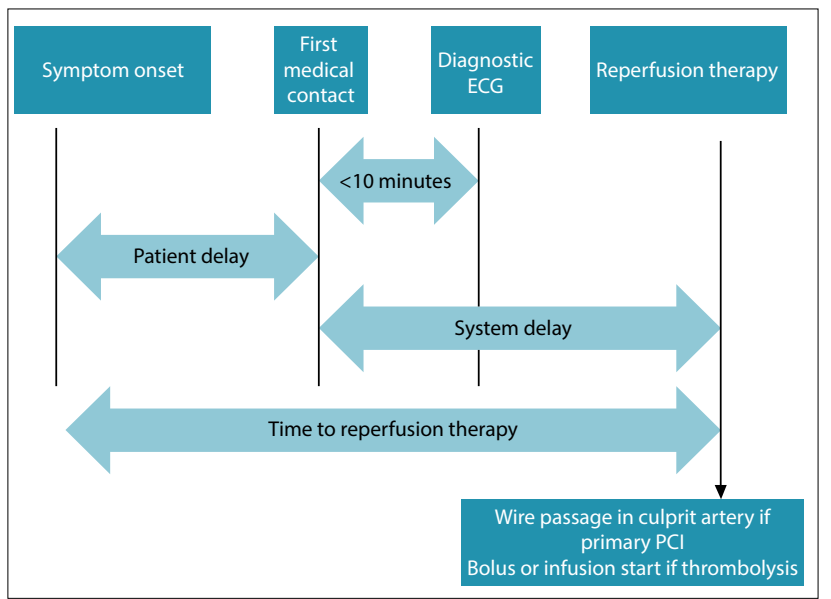

Fig. 3. Components in the delays from first medical contact to reperfusion therapy for STEMI patients.

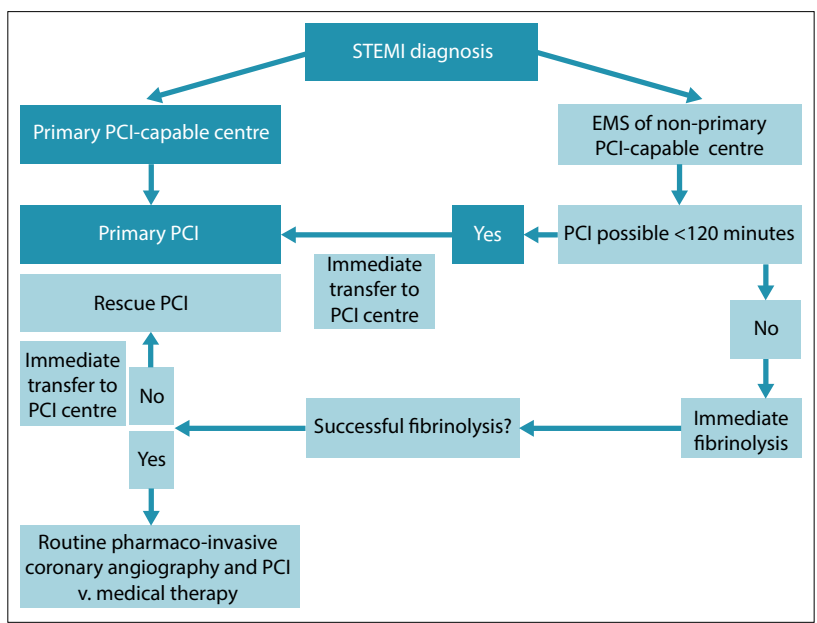

Fig. 4. Prehospital and inpatient management of STEMI (EMS = emergency medical services).

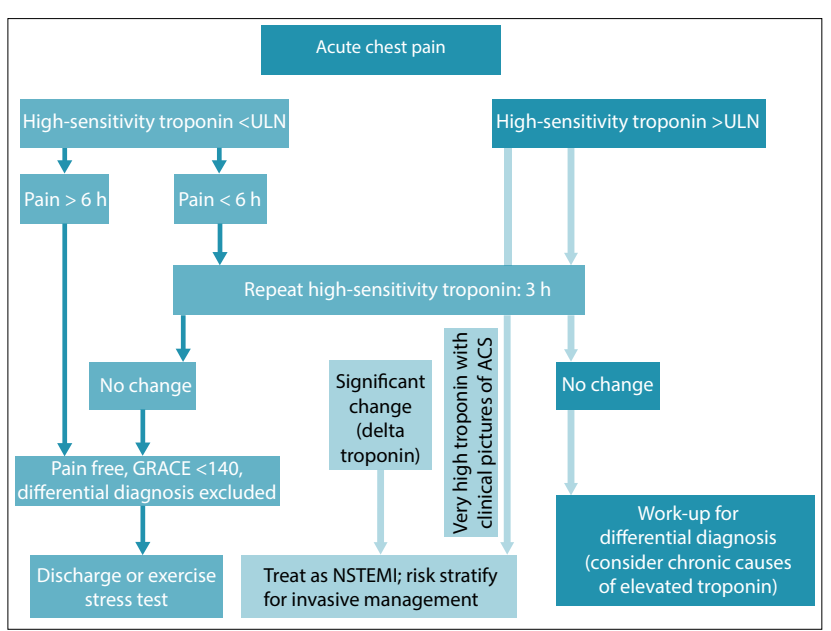

Fig. 5. Rapid rule-out of ACS using high-sensitivity troponin (ULN = upper limit of normal).

\section{Less common ACS aetiologies}

Infrequently, aetiologies other than ruptured atheroma result in ACS. Examples include coronary artery embolisation (in patients with atrial fibrillation, cardiomyopathy with ventricular clot or septic emboli from infective endocarditis), spontaneous coronary dissection (in patients with fibromuscular dysplasia, coronary vasculitis or thyrotoxicosis), coronary artery spasm (recreational
Table 4. Contraindication to fibrinolysis

\begin{tabular}{ll}
\hline Absolute & Relative \\
\hline $\begin{array}{l}\text { Previous intracranial } \\
\text { haemorrhage or stroke of } \\
\text { unknown origin at any time }\end{array}$ & $\begin{array}{l}\text { Transient ischaemic attack in } \\
\text { the preceding } 6 \text { months }\end{array}$ \\
$\begin{array}{l}\text { Ischaemic stroke in the preceding } \\
6 \text { months }\end{array}$ & Oral anticoagulation therapy \\
$\begin{array}{l}\text { Central nervous system damage } \\
\text { or neoplasm or atrioventricular } \\
\text { malformations }\end{array}$ & $\begin{array}{l}\text { Pregnancy or within 1 week } \\
\text { post partum }\end{array}$ \\
$\begin{array}{l}\text { Recent major trauma/surgery/ } \\
\text { head injury (within preceding }\end{array}$ & $\begin{array}{l}\text { Refractory hypertension } \\
\text { (systolic BP }>180 \text { mmHg }\end{array}$ \\
$\begin{array}{l}\text { and/or } \\
\text { diastolic BP }>110 \text { mmHg) }\end{array}$ \\
$\begin{array}{l}\text { Gastrointestinal bleeding within } \\
\text { the past month }\end{array}$ & $\begin{array}{l}\text { Advanced liver disease } \\
\text { Known bleeding disorder } \\
\text { (excluding menses) }\end{array}$ \\
$\begin{array}{l}\text { Aortic dissection } \\
\text { Non-compressible puncture } \\
\text { in the past 24 hours (e.g. liver } \\
\text { biopsy, lumbar puncture) }\end{array}$ & $\begin{array}{l}\text { Infective endocarditis } \\
\text { Prolonged or traumatic }\end{array}$ \\
\end{tabular}

drug use, de novo) and stress-induced cardiomyopathy (Takotsubo non-ischaemic cardiomyopathy). Careful history and clinical examination can often identify these patients prior to definitive ACS care. Even so, the general principles of management are as outlined above.

\section{ACSs in sub-Saharan Africa}

ACSs are becoming increasingly prevalent in sub-Saharan Africa, with patients displaying similar myocardial risk factors to those of the developed world. ${ }^{[1,17]}$ There are limited data regarding ACSs in the SA context, with observational data suggesting that there are high intervention rates and frequent use of evidence-based pharmacological therapies with comparative outcomes when compared with international standards. ${ }^{[18]}$ These data reflect mainly the care delivered in the private sector, and do not fully represent care from the public sector where resources are limited and timely presentation to healthcare facilities capable of managing ACSs is often delayed. ${ }^{[10,16]}$ Our current challenge is the provision of equal access and quality ACS care to all sectors of the SA population. This would entail reliance on the pharmaco-invasive strategy (routine and rescue) until there is adequate infrastructure expansion to accommodate the roll-out of a comprehensive, organised, hub-and-spoke PPCI service. Initiatives such as the European Society of Cardiology 'Stent-for-Life' project and planned national ACS registries could be the stepping stones to formalising management of ACSs on a national level.

\section{References}

1. Bösner S, Becker A, Haasenritter J, et al. Chest pain in primary care: Epidemiology and pre-work-up probabilities. Eur J Gen Pract 2009;15(3):141-146. [http://dx.doi.org/10.3109/13814780903329528] 2. Kramer L, Rabanizada N, Haasenritter J, Bösner S, Baum E, Donner-Banzhoff N. Do guidelines on firs impression make sense? Implementation of a chest pain guideline in primary care: A systematic evaluation of acceptance and feasibility. BMC Family Practice 2011;12:128. [http://dx.doi.org/10.1186/1471-2296-12-128]

3. Anderson JL, Adams CD, Antman EM, et al. ACC/AHA 2007 guidelines for the management of patients with unstable angina/non-ST-elevation myocardial infarction: Executive summary. Circulation 2007;116(7):803-877. [http://dx.doi.org/10.1161/CIRCULATIONAHA.107.185752]

4. Haasenritter J, Bösner S, Vaucher P, et al. Ruling out coronary heart disease in primary care: External validation of a clinical prediction rule. Br J Gen Pract 2012;62(599):415-421. [http://dx.doi. org/10.3399/bjgp12X649106]

5. Antman EM, Tanasijevic MJ, Thompson B, et al. Cardiac-specific troponin I levels to predict the risk of mortality in patients with acute coronary syndromes. N Engl I Med 1996;335(18):1342-1349. [http:// dx.doi.org/10.1056/NEJM199610313351802] 
Table 5. TIMI, GRACE and CRUSADE risk scores

\section{TIMI risk score for UA/NSTEMI}

\begin{tabular}{|c|c|c|c|c|}
\hline \multirow[b]{2}{*}{ Variable } & \multirow[b]{2}{*}{ Points } & \multicolumn{3}{|c|}{ Risk of cardiac events (\%) by 14 days } \\
\hline & & Risk score & Death or MI & $\begin{array}{l}\text { Death, MI or urgent } \\
\text { revascularisation }\end{array}$ \\
\hline Age $\geq 65$ years & 1 & $0-1$ & 3 & 5 \\
\hline $\begin{array}{l}\geq 3 \text { CAD risk factors (family history, raised cholesterol, diabetes, } \\
\text { hypertension) }\end{array}$ & 1 & 2 & 3 & 8 \\
\hline Known CAD (stenosis $\geq 50 \%$ ) & 1 & 3 & 5 & 13 \\
\hline Aspirin use in past 7 days & 1 & 4 & 7 & 20 \\
\hline Recent severe angina (last 24 hours) & 1 & 5 & 12 & 26 \\
\hline Elevated cardiac biomarkers & 1 & $6-7$ & 19 & 41 \\
\hline ST deviation $\geq 0.5 \mathrm{~mm}$ & 1 & & & \\
\hline
\end{tabular}

\section{GRACE score for UA/NSTEM}

\section{Variables*}

Age

\section{Heart failure}

Peripheral vascular disease

Admission systolic BP

Killip class

Elevated cardiac biomarkers

Initial serum creatinine

Cardiac arrest on admission

ST segment deviation

*Variables are inserted into an online calculator to generate the GRACE score

* Variables are inserted
(www.gracescore.org)

\section{CRUSADE bleeding risk score}

\section{Variables}

Baseline haematocrit

Estimated glomerular filtration rate

Heart rate on admission

Systolic BP on admission

Prior vascular disease

\begin{tabular}{lc}
\multicolumn{2}{c}{ In-hospital mortality } \\
\hline Risk categories & GRACE score $\quad$ Death, $\%$ \\
\hline
\end{tabular}

$\begin{array}{lll}\text { Low } & 1-108 & <1 \\ \text { Intermediate } & 109-140 & 1-3 \\ \text { High } & 141-372 & >3\end{array}$

6 months post-discharge mortality

\begin{tabular}{lll}
\hline Risk categories & GRACE score & Death, \% \\
\hline Low & $1-88$ & $<3$ \\
Intermediate & $89-118$ & $3-8$ \\
High & $119-263$ & $>8$
\end{tabular}

Diabetes mellitus

Signs of congestive heart failure on admission

Gender

${ }^{\dagger}$ Variables are inserted into an online calculator to generate the CRUSADE score (www.crusadebleedingscore.org/).

6. Reichlin T, Hochholzer W, Bassetti S, et al. Early diagnosis of myocardial infarction with sensitive cardiac troponin assays. N Engl J Med 2009;361(9):858-867. [http://dx.doi.org/10.1056/NEJMoa0900428]

7. Thygesen K, Alpert JS, Jaffe AS, et al. Third universal definition of myocardial infarction. J Am Coll Cardiol 2012;60(16):1581-1598. [http://dx.doi.org/10.1016/.j.jacc.2012.08.001]

8. Hamm CW, Bassand J-P, Agewall S, et al. ESC guidelines for the management of acute coronary syndromes in patients presenting without persistent ST-segment elevation: The Task Force for the management of acute coronary syndromes (ACS) in patients presenting without persistent ST-segment elevation. Eur Heart J 2011;32(23):2999-3054. [http://dx.doi.org/10.1093/eurhearti/ehr236]

9. Steg PG, James SK, Atar D, et al. ESC guidelines for the management of acute myocardial infarction in patients prestingwith ST presenting myocardial infarction in Cape Town. S Afr Med J 2012;102(4):241-244.

. Delport R. Towards developing guidelines and systems of care to facilitate early reperfusion for ST(256-258.

12. Harker M, Carville S, Henderson R, Gray H. Key recommendations and evidence from the NICE guideline for the acute management of ST-segment-elevation myocardial infarction. Heart 2014;100(7):536-543. [http://dx.doi.org/10.1136/heartjnl-2013-304717]

13. Lubitz J, Riley G. Trends in medicare payments in the last year of life. N Engl J Med 1993;328(15):1092 1096. [http://dx.doi.org/10.1056/NEJM199304153281506]
14. Assessment of the Safety and Efficacy of a New Thrombolytic (ASSENT-2) Investigators, van de Werf F, Adgey J, et al. Single-bolus tenecteplase compared with front-loaded alteplase in acute myocardial infarction: The ASSENT-2 double-blind randomised trial. Lancet 1999;354(9180):716-722.

15. Welsh RC, van de Werf F, Westerhout CM, et al. Outcomes of a pharmacoinvasive strategy for successful versus failed fibrinolysis and primary percutaneous intervention in acute myocardial infarction (from the Strategic Reperfusion Early After Myocardial Infarction [STREAM] Study). Am J Cardiol 2014;114(6):811-819. [http://dx.doi.org/10.1016/j.amjcard.2014.06.011]

16. Shavadia J, Yonga G, Otieno H. A prospective review of acute coronary syndromes in an urban hospital in sub-Saharan Africa. Cardiovasc J Afr 2012;23(6):318-321. [httt://dx.doi.org/10.5830/CVJA-2012-002]

17. Ntsetse infarction and stroke in sub-Saharan Africa. Heart 2013;99(17):1230-1235. [http://dx.doi.org/10.1136/
inter. heartinl-2012-303585]

18. Schamroth C. Management of acute coronary syndrome in South Africa: Insights from the ACCESS (Acute Coronary Events - a Multinational Survey of Current Management Strategies) registry: Cardiovascular topics. Cardiovasc J Afr 2012;23(7):365-370. [http://dx.doi.org/10.5830/ CVJA-2012-017]

19. De Winter RJ, Verouden NJW, Wellens HJJ, Wilde AM. A new ECG sign of proximal LAD occlusion. N Engl J Med 2008;359(19):2071-2073. [http://dx.doi.org/10.1056/NEJMc0804737] 